\title{
The effects of smoking on quality of life recovery after surgery for chronic rhinosinusitis*
}

\section{Michael Katotomichelakis', Efthimios Simopoulos' ${ }^{1}$ Gregory Tripsianis², Nan Zhang ${ }^{3}$, Gerasimos Danielides ${ }^{1}$, Panagiota Gouma', Claus Bachert ${ }^{3}$, Vassilios Danielides ${ }^{1}$}

Rhinology 52: 341-347, 2014

DOl:10.4193/Rhino13.231

*Received for publication:

December 30, 2013

Accepted: March 23, 2014

2 Department of Statistics, Medical School, Democritus University of Thrace, Alexandroupolis, Evros, Greece

${ }^{3}$ Upper Airway Research Laboratory, Department of Otorhinolaryngology, Ghent, University Hospital, Ghent, Belgium

\begin{abstract}
Background: The effects of smoking on quality of life (QoL) results in chronic rhinosinusitis (CRS) patients still remain a matter of debate.

Objective: To explore the impact of smoking on QoL and determine how quantity of daily smoking and duration affect QoL results after endoscopic sinus surgery (ESS).

Methodology: Patients with CRS were prospectively asked to evaluate their QoL pre-and after ESS. All subjects' QoL was evaluated by means of validated questionnaires either specific (Questionnaire of Olfactory Deficits), for assessing psychology (Zung Anxiety Scale, State-Trait Anxiety Inventory, Zung Depression Scale and Beck Depression Inventory) or generic (Short Form-36). Smoking habits were expressed in pack-years.
\end{abstract}

Results: No statistically significant differences were found in the pre-treatment scores in any of the questionnaires between smokers and non smokers. Post-operatively, all QoL questionnaires' results were significantly improved among both groups, although non smokers exhibited significantly greater improvement compared to smokers. There was a negative impact of the number of pack-years on the changes of QoL results; less improvement was observed as the number of pack-years was increasing.

Conclusion: Although smoking did not influence preoperative QoL results and proved not to be a contra-indication factor for ESS regarding QoL, smokers presented worse treatment outcomes. Quantity and duration of smoking were significantly associated with worse postoperative results in all QoL questionnaires.

Key words: quality of life, chronic rhinosinusitis, smoking, quantity and duration of smoking, endoscopic sinus surgery

\section{Introduction}

Smoking is generally considered a risk factor with hazardous effects on upper airway diseases ${ }^{(1-5)}$, and is accepted as a predisposing factor that is closely related to chronic rhinosinusitis $(\mathrm{CRS})^{(6-8)}$. Despite the negative effects of smoking on sinonasal mucosa ${ }^{(4,5)}$, the literature regarding endoscopic sinus surgery (ESS) outcomes in smokers versus non smokers is conflicting ${ }^{(9-20)}$. Moreover, the effects of smoking on quality of life (QoL) results in CRS patients either pre-operatively or after surgery is still a matter of debate ${ }^{(11-18)}$.

This means that questions such as how smoking is correlated to QoL results in CRS patients, and if smokers are good candidates or not for ESS according to their QoL outcomes have not been adequately addressed in literature and remain without a clear answer. Additionally, the correlation between quantity and duration of smoking (pack-years) and ESS outcomes with regard to QoL results has not been evaluated and needs to be further explored ${ }^{(1,6)}$. The clinical importance is that this data may 
prove valuable for otolaryngologists either in supporting clinical decisions on the optimal management of smokers suffering from CRS, facilitating case selections or in counselling patients about the anticipated benefit for their QoL after ESS and how it is correlated to their smoking habits.

Accordingly, the aims of the present study were to explore the effects of smoking on CRS patients' QoL pre-treatment, to prospectively evaluate the overall effect of smoking on postoperative outcomes (quality-of-life) and to determine if quantity of daily smoking and duration, expressed in pack-years, affect QoL outcomes.

\section{Materials and methods}

\section{Human subjects}

One hundred eleven patients suffering from chronic rhinosinusitis (either with nasal polyps: CRSwNP, or without NP: CRSsNP) were studied. Diagnosis was based on history, clinical examination, nasal endoscopy, sinus computed tomography scanning (CT), skin prick testing (SPT) for atopy, test of pulmonary function and olfactory testing ${ }^{(21,22)}$. CT scans were graded according to the Lund-MacKay scoring system ${ }^{(23)}$. Forty-eight out of 111 (43.2\%) patients had a total score of $>16$ (range, 17-24); there was bilateral ethmoid disease with involvement of 3 or more dependent sinuses and of the ostiomeatal complex. Twenty four (21.6\%) patients presented with mild CT scan appearance with a total score of $<9$ (range, 1-8), and 49 (44.1\%) patients had a CT total score that ranged between 9 to 16 . All patients fulfilled the criteria of CRS according to the EPOS (24) guidelines. The smokers' cohort included patients who smoked at the time of surgery and there was no change on their smoking habits after ESS; whereas those who smoked only in the past were excluded, because the impact of prior smoking may be a confusing factor on results. Non smokers were all these patients who declared they never smoked in their lives. Smoking habits were measured in pack per years, calculated by the number of packs smoked per day multiplied by the number of years smoking. All subjects underwent a brief psychiatric interview to exclude those with a pre-existing major psychiatric disorder.

\section{Treatment procedures}

ESS was the treatment of choice after failing maximal medical therapy (antibiotics, oral and nasal steroids) for at least six months before surgery. It was conducted by a single surgeon following the Messerklinger ${ }^{(25)}$ technique according to the extent of the disease, as provided by CT scans score ${ }^{(23)}$, with the use of a microdebrider device. Septoplasty and inferior nasal turbinates (INT) volume reduction- by submucosal radiofrequency tissue ablation- were implemented whenever significant septal deviation or INT enlargement were observed. Post-operative care adjuvant procedures were the same for both groups and included multiple visits at the outpatient clinic where nasal endoscopy and cleaning of crusts and debris, lysis of adhesions and removal of any single polypoid mass were performed. All patients were asked postoperatively to rinse the nose with sodium chloride solution and nasal steroids.

The study protocol was approved by the local Institutional Review Board. All subjects signed informed consent. The study was performed in accordance with the Declaration of Helsinki/ Hong Kong.

\section{QoL assessment}

Patients' QoL assessment was achieved by means of validated, widely used questionnaires either specific for olfaction-associated QoL (Questionnaire of Olfactory Deficits-QOD) and for delineating mental health (Zung Anxiety Scale-ZAS, State and Trait Anxiety Inventory-STAI, Beck Depression Inventory-BDI and Zung Depression Scale-ZDS) or using a general health survey (Short Form -SF-36).

QOD represents an olfaction-specific questionnaire for QoL, translated and validated for the Greek population ${ }^{(26)}$. It was used to investigate the independent effect of smoking on QoL results irrespective of the improvement of the olfactory function. It consists of 25 4-scale statements (17 "negative", 2 "positive", 6 "socially desired") with a maximum score of 57 points (26). High scores indicate a strong impairment in QoL. Anxiety symptoms were assessed by means of ZAS (27) and STAI ${ }^{(28)}$ scales also translated and validated in Greek. ZAS is a self-rating scale which explores levels of anxiety ${ }^{(27)}$. STAI is an instrument consisting of two parts, one that refers to anxiety due to a specific condition (state), and one that refers to the general tendency of the subject to react anxiously (trait) ${ }^{(28)}$. Each one of these parts has 20 questions, which are answered by choosing between grades 1 to 4. Depression was assessed using the Greek validated versions of $\mathrm{BDI}{ }^{(29)}$ and $\mathrm{ZDS}{ }^{(30)}$. BDI consists of 21 self reporting items graded from 0 to 3, corresponding to the level of depressive symptomatology and a higher score indicates higher level of depressive mood ${ }^{(29)}$. ZDS ${ }^{(30)}$ is another self-administered instrument with a structure similar to ZAS, measuring depression. It consists of 20 items graded from 1 to 4 and assesses psychological and somatic depression. Higher scores indicate higher level of depression. Finally, SF-36 is a widely used general health related survey that assesses QoL in eight domains covering both physical and mental health from the patient's point of view with chronic diseases ${ }^{(31,32)}$. Scores range from 0 to 100 , with a higher score representing better functioning ${ }^{(31,32)}$.

\section{Statistics}

Statistical analysis of the data was performed using the Statistical Package for the Social Sciences (SPSS), version 19.0 (IBM). The normality of quantitative variables was ascertained with Kolmogorov-Smirnov test. Normally distributed quantitative 
variables were expressed as mean \pm standard deviation (SD), while non-normally distributed variables were expressed as median and interquartile range (IQR, 25th to 75th percentile); quantitative variables were expressed as frequencies and percentages (\%). The chi-square test, Student's t test and MannWhitney U-test were used to assess differences of demographic and disease characteristics between smokers and non smokers. One-way analysis of covariance (ANCOVA) was performed to investigate the effect of smoking on (i) pre-treatment scores and (ii) post-treatment changes of the scores of QoL questionnaires, adjusting for all potential confounders. Since the distribution of BDI score was skewed, the statistical analysis was performed on the log-transformed scores. The effect size (ES) of smoking habits on the changes of the scores of quality of life (QoL) questionnaires of chronic rhinosinusitis patients 12-months after treatment was described in terms of Cohen's d. An ES of 0.2 to 0.3 represents a "small" effect, around 0.5 a "moderate" effect and 0.8 to infinity, a "large" effect ${ }^{(33)}$. Spearman's $\rho$ correlation coefficient was used to assess the relation between pack-years and the post-treatment changes of the scores of QoL questionnaires. To assess the independent effect of the number of pack-years on the post-operative values of the QoL questionnaires multivariate linear regression analysis were constructed. All tests were two tailed and statistical significance was considered for $p$ values of less than 0.05 .

\section{Results}

\section{Patients' characteristics}

The study group included one hundred eleven patients with a mean age of $44.74 \pm 16.21$ years (range, $14-71$; median age 45.5 years). Fifty-two (46.8\%) of them were females, with a mean age of $50.60 \pm 15.22$ years and 59 (53.2\%) were males, with mean age $39.58 \pm 15.39$ years. A history of current smoking was reported in 48 (43.2\%) patients, while 63 patients (56.8\%) had never smoked. The group of smokers consisted of 21 females (43.8\%) and 27 (56.3\%) males, (mean age $37.98 \pm 13.21$ years; range, 21-59 years). The duration of smoking ranged from 4 to 36 years, with a median duration of 12 years (IQR, 10-30 years); moreover, the number of packs smoked per day ranged from 0.25 to 4 packs, with a median value of 1 pack per day (IQR, 0.51.5 packs per day). The group of non smokers consisted of 31 (49.2\%) females and 32 (50.8\%) males (mean age $44.60 \pm 17.74$ years; range, 14-71 years). Demographics and disease characteristics of the patients in relation to smoking habits are presented in Table 1. There were no statistically significant differences in gender $(p=0.568)$ and socio-economic status $(p=0.180)$ between smokers and non smokers; on the contrary, smokers were significantly younger than non smokers $(p=0.026)$. Allergic rhinitis ( $58.7 \%$ vs $31.3 \%, p=0.004)$, asthma $(27.0 \%$ vs $0.0 \%$, $p$ $<0.001)$ and aspirin intolerance $(15.9 \%$ vs $4.2 \%, p=0.049)$ were more frequent among non smokers than smokers; the frequen-
Table 1. Demographics and disease characteristics of chronic rhinosinusitis patients in relation to their smoking habits.

\begin{tabular}{|c|c|c|c|}
\hline & $\begin{array}{l}\text { Non-smokers } \\
\qquad(n=63\end{array}$ & $\begin{array}{l}\text { Smokers } \\
(n=48)\end{array}$ & p value \\
\hline Male gender & $32(50.8)$ & $27(56.2)$ & 0.568 \\
\hline Age (years) & 47.60 (17.74) & $40.98(13.21)$ & 0.026 \\
\hline Socio-economic status & & & 0.180 \\
\hline Low & $6(9.5)$ & $2(4.2)$ & \\
\hline Medium & $32(50.8)$ & $19(39.6)$ & \\
\hline High & $25(39.7)$ & $27(56.2)$ & \\
\hline $\begin{array}{l}\text { Duration of smoking } \\
{[\min / \max , 4 / 35]}\end{array}$ & - & $12(10-30)$ & - \\
\hline \multicolumn{4}{|l|}{ Disease characteristics } \\
\hline Allergic rhinitis & $37(58.7)$ & $15(31.3)$ & 0.004 \\
\hline Asthma presence & $17(27.0)$ & $0(0.0)$ & $<0.001$ \\
\hline Nasal polyps & $29(46.0)$ & $25(52.1)$ & 0.527 \\
\hline Aspirin intolerance & $10(15.9)$ & $2(4.2)$ & 0.049 \\
\hline CT scan score & & & 0.844 \\
\hline$<9$ & $13(20.6)$ & $11(22.9)$ & \\
\hline $9-16$ & $27(42.9)$ & $22(45.8)$ & \\
\hline$>16$ & $23(36.5)$ & $15(31.3)$ & \\
\hline TDI score & $19.31(9.12)$ & $28.19(10.36)$ & $<0.001$ \\
\hline Olfactory function & & & 0.001 \\
\hline Normosmics & $9(14.3)$ & $18(37.5)$ & \\
\hline Hyposmics & $25(39.7)$ & $23(47.9)$ & \\
\hline Anosmics & $29(46.0)$ & 7 (14.6) & \\
\hline $\begin{array}{l}\text { Duration of olfactory } \\
\text { dysfunction (years) }\end{array}$ & $10(5-15)$ & $5(2-8.5)$ & 0.01 \\
\hline
\end{tabular}

Normally distributed quantitative variables were expressed as mean (standard deviation, SD); non-normally distributed quantitative variables were expressed as median (interquartile range, IQR); qualitative variables were expressed as frequencies (percentage, \%).

cy of nasal polyps was similar in the two group of patients $(\mathrm{p}=$ 0.527). Similarly, the severity of the disease as expressed by CT scan score was comparable between smokers and non-smokers $(p=0.581)$ and was not correlated with the quantity of smoking (pack-years) (spearman's $\rho=0.198, p=0.207$ ). Also there was not any significant association between pack-years and age ( $\rho$ $=0.077, p=0.375$ ). Regarding to the quantitative assessment 
Table 2. Pre-treatment scores of quality of life (QoL) questionnaires of chronic rhinosinusitis patients in relation to smoking habits. Data are expressed as adjusted mean values (standard error, SE).

\begin{tabular}{|c|c|c|c|c|}
\hline & $\begin{array}{l}\text { Non- } \\
\text { smokers }\end{array}$ & Smokers & $\begin{array}{l}\text { Mean difference } \\
\qquad(95 \% \mathrm{CI})\end{array}$ & p value \\
\hline SF-36 & $68.52(1.81)$ & $67.78(2.14)$ & $0.74(-5.43$ to 6.90$)$ & 0.813 \\
\hline QOD & $17.71(1.07)$ & $16.34(1.26)$ & $1.37(-2.27$ to 5.01$)$ & 0.456 \\
\hline QOD-NS & $10.42(0.98)$ & $8.68(1.16)$ & $1.73(-1.62$ to 5.08$)$ & 0.308 \\
\hline QOD-PS & $4.53(0.15)$ & $4.85(0.18)$ & $-0.32(-0.78$ to 0.14$)$ & 0.172 \\
\hline QOD-SD & $2.97(0.20)$ & $2.69(0.24)$ & $0.28(-0.41$ to 0.97$)$ & 0.426 \\
\hline Zung Anxiety Scale & $40.87(1.07)$ & $39.67(1.26)$ & $1.20(-2.46$ to 4.86$)$ & 0.517 \\
\hline STAI & $44.87(0.92)$ & 44.08 (1.09) & $0.79(-2.35$ to 3.94$)$ & 0.618 \\
\hline Zung Depression Scale & $39.61(1.09)$ & $38.56(1.29)$ & 1.05 (-2.67 to 4.76$)$ & 0.577 \\
\hline Beck Depression Inventory & $12.25(0.94)$ & $12.67(1.11)$ & $-0.42(-3.61$ to 2.77$)$ & 0.795 \\
\hline
\end{tabular}

of subjects' olfactory function with the use of Sniffin' Sticks test, non smokers presented statistically significant lower "ThresholdDiscrimination-Identification(TDI)" score compared to smokers (19.31 \pm 9.12 vs $28.19 \pm 10.36, p<0.001)$.

\section{Pre-treatment results}

Since there were significant differences in demographic and disease characteristics between smokers and non smokers, analysis of covariance was performed to investigate the effect of smoking on patients' QoL, controlling for the effect of all possible confounders. The adjusted pre-treatment scores of all QoL questionnaires of smokers and non smokers are shown in Table 2. There was no statistically significant difference in the pretreatment scores in any of the questionnaires between smokers and non smokers (SF-36: $p=0.813 ;$ QOD: $p=0.456$; ZAS: $p=$ $0.517 ;$ STAI: $p=0.618 ;$ ZDS: $p=0.577 ; B D I: p=0.795$ ).

\section{Post-operative results}

Throughout the 12-month follow up time, a significant improvement of the scores of all QoL questionnaires (i) was observed among non smokers: SF-36 by $28.3 \%(\mathrm{p}<0.001)$, QOD by $-59.9 \%(p<0.001)$, ZAS by $-28.9 \%(p<0.001)$, STAI by $-24.9 \%$ $(p<0.001)$, ZDS by $-24.7 \%(p<0.001)$ and BDI by $-36.1 \%(p$ $<0.001)$ and (ii) among smokers: SF-36 by $21.1 \%(p<0.001)$, QOD by $-37.9 \%(p<0.001)$, ZAS by $-17.6 \%(p<0.001)$, STAI by $-10.8 \%(p<0.001)$, ZDS by $-14.5 \%(p<0.001)$ and BDI by $-10.8 \%(p<0.001)$. The adjusted changes of the scores of all QoL questionnaires of smokers and non smokers are shown in Table 3. The comparison of these changes between smokers and non smokers, controlling for the effect of all patients' characteristics, revealed that non smokers exhibited significantly greater improvement of all QoL questionnaires compared to smokers
(SF-36: $p=0.046 ;$ QOD: $p=0.032 ;$ ZAS: $p<0.001 ;$ STAI: $p<0.001$; ZDS: $p=0.001 ; B D I: p=0.036)$.

\section{Quantity/duration of smoking and QoL outcomes}

To assess the effect of the quantity and duration of smoking on the improvement of QoL questionnaires, the number of packyears was calculated for each smoker, based on the number of packs smoked per day and the number of years that smoking occurred. The number of pack-years ranged from 1.5 to 60.0, with a median number of 10 pack-years (IQR, 5.25-20). In correlation analysis between the changes of QoL questionnaires and the number of pack-years using Spearman's $\rho$ correlation coefficient (Table 4), there was a tendency towards smaller improvement of smokers' QoL as the number of pack-years was increasing (SF-36: $\rho=-0.391, p=0.006$; QOD: $\rho=0.392, p=$ 0.009; ZAS: $\rho=0.349, p=0.015$; STAl: $\rho=0.410, p=0.006$; ZDS: $\rho=0.317, p=0.028$; BDI: $\rho=0.263, p=0.071$ ). In particular, multivariate linear regression analysis on the post-operative values of the QoL questionnaires showed that an increase of 10 in pack-years was ensued by a significant increment of QOD by 2.00 points $(p=0.003)$, ZAS by $8.28(p=0.005)$, STAI by $9.11(p=$ $0.001)$, ZDS by $8.87(p=0.006)$, BDI by $1.65(p=0.034)$ and a decrease of SF-36 by 12.35 points $(p<0.001)$, which indicates the deterioration of all post-operative QoL questionnaires results as the number of pack-years increases.

\section{Discussion}

This study provides evidence for the effects of smoking habits on CRS patients' QoL and explores whether smokers experience poorer outcomes after ESS with regard to QoL results, based on reliable, validated, widely used questionnaires for QoL assessment. We didn't include more than QOD disease specific quality 
Table 3. Changes of the scores of quality of life (QoL) questionnaires of chronic rhinosinusitis patients 12-months after treatment in relation to smoking habits. Data are expressed as adjusted mean values (standard error, SE).

\begin{tabular}{|c|c|c|c|c|c|}
\hline & $\begin{array}{l}\text { Non- } \\
\text { smokers }\end{array}$ & Smokers & $\begin{array}{l}\text { Mean difference } \\
(95 \% \mathrm{Cl})\end{array}$ & p value & Cohen's d \\
\hline SF-36 & $19.39(1.55)$ & $14.29(1.81)$ & 5.10 (0.10 to 10.10$)$ & 0.046 & 0.41 \\
\hline QOD & $-10.61(1.22)$ & $-6.20(1.43)$ & $-4.41(-8.44$ to -0.39$)$ & 0.032 & 0.45 \\
\hline QOD-NS & $-9.60(0.99)$ & $-6.44(1.14)$ & $-3.16(-6.19$ to -0.13$)$ & 0.041 & 0.40 \\
\hline QOD-PS & $1.37(0.16)$ & $0.60(0.19)$ & 0.77 (0.24 to 1.29$)$ & 0.005 & 0.62 \\
\hline QOD-SD & $-1.63(0.21)$ & $-1.34(0.24)$ & $-0.29(-0.92$ to 0.35$)$ & 0.375 & 0.17 \\
\hline Zung Anxiety Scale & $-11.82(0.78)$ & $-6.97(0.92)$ & $-4.85(-7.52$ to -2.18$)$ & $<0.001$ & 0.77 \\
\hline STAI & $-11.18(0.81)$ & $-4.78(0.97)$ & $-6.40(-9.17$ to -3.63$)$ & $<0.001$ & 0.97 \\
\hline Zung Depression Scale & $-9.79(0.74)$ & $-5.59(0.88)$ & $-4.20(-6.72$ to -1.68$)$ & 0.001 & 0.70 \\
\hline Beck Depression Inventory & $-4.42(0.44)$ & $-1.37(1.28)$ & $-3.05(-5.91$ to -0.20$)$ & 0.036 & 0.47 \\
\hline
\end{tabular}

of life instruments as we would like to provide general conclusions that could be used either in every day clinical practice or in future studies to allow comparisons between different diseases, conditions or treatments. However, it should be useful in future studies the combination of generic and more disease specific QoL measures for monitoring changes in a patient's QoL due to an intervention, as well. To the best of our knowledge this is the first study to investigate the impact of quantity of daily smoking and duration of smoking, expressed in pack-years, on QoL outcomes. Moreover, we identified the increase in the number of pack-years that influences significantly QoL questionnaires results. The results of this study follow our recent work

Table 4. Association between the changes of quality of life (QoL) questionnaires and pack-years, expressed as Spearman's $\rho$ correlation coefficient.

\begin{tabular}{|c|c|c|}
\hline & Pack years & p value \\
\hline$\Delta S F-36_{0,12}$ & -0.391 & 0.006 \\
\hline$\Delta \mathrm{QOD}_{0,12}$ & 0.392 & 0.009 \\
\hline$\Delta Q O D-N_{0,12}$ & 0.298 & 0.040 \\
\hline$\triangle \mathrm{QOD}-\mathrm{PS}_{0,12}$ & -0.279 & 0.055 \\
\hline$\Delta \mathrm{QOD}^{-\mathrm{SD}_{0,12}}$ & 0.224 & 0.126 \\
\hline$\Delta$ Zung Anxiety Scale ${ }_{0,12}$ & 0.349 & 0.015 \\
\hline$\Delta$ State and Trait Anxiety Inventory ${ }_{0,12}$ & 0.410 & 0.006 \\
\hline$\Delta$ Zung Depression Scale ${ }_{0,12}$ & 0.317 & 0.028 \\
\hline$\Delta$ Beck Depression Inventory $_{0,12}$ & 0.263 & 0.071 \\
\hline
\end{tabular}

describing the negative effects of smoking on olfaction ${ }^{(5)}$, but now we focus on QoL rehabilitation in CRS patients, adding new knowledge to the issue of smoking/QoL interaction that is not yet well delineated and worth pursuing further study ${ }^{(1,6)}$. First, we explored if smoking affects CRS patients' QoL, pretreatment. Since in the pre-operative assessment, there was a statistically significant difference in some disease characteristics (pre-operative prevalence of asthma, allergic rhinitis and ASA intolerance in non smokers), we have to point out that using appropriate statistical methods, we investigated the effect of smoking on patients' QoL, controlling for the effect of all possible confounders. According to the adjusted pre-treatment scores of all QoL questionnaires' outcomes, no difference was found in pre-treatment scores of all QoL questionnaires between smokers and non smokers. This means that there is no impact of smoking habits as well as its duration on pre-operative QoL results. It is interesting to mention that smokers were significantly younger, possibly indicating that smokers develop CRS earlier in life, providing evidence of the close relation of smoking to CRS $(4,5)$.

The important question that is raised now is if active smoking status should be considered a contra-indication or not to ESS because of a concern for or perception of poor surgical outcomes. In our study, we found that both smoker and nonsmoker patients presented a significant improvement on all QoL questionnaires' results. Our findings suggest that smoking status should not be considered a contra-indication to ESS for these patients, and there is no selection limits for ESS related to smoking habits. However, the comparison of these changes between smokers and non smokers, controlling for the effect of all patients' characteristics, revealed that the improvement in non smokers was significantly higher than in smokers, ac- 
cording to all QoL questionnaire results. Complementary to the statistical significance ( $p$-values), either the upper or lower limits of the $95 \% \mathrm{Cl}$ of the QoL changes between non-smokers and smokers (i.e. 10.10 points change in SF-36, -5.91 points in $\mathrm{BDI}$, etc.) or the Cohen's d effect size, both indicate moderate to large effect of smoking in changes of QoL ${ }^{(33)}$. Moreover, it is also important to mention that based on our recent work ${ }^{(34)}$ that defined clinically significant improvement in QoL of CRS patients, using methodology as provided by Norman et al. (35), we observed that mean differences in changes of the scores of QoL questionnaires among groups after ESS except of statistical significance, represent a clinical significance for patients, as well. This is in accordance with previous studies ${ }^{(36-38)}$ that defined clinically meaningful differences in the results of the QoL instruments used. Thus, although active smoking status proved not to be a contra-indication to ESS with regard to QoL outcomes, our findings support clinical decisions on counselling patients to quit smoking, in addition to many other health reasons, for the possibility of poorer post-surgery outcomes if they should continue smoking. In our study, we counselled patients not to change their smoking habits post-operatively, as we would like to investigate the effects of ESS on smokers related to post-operative $Q$ oL results and not the effects of smoking cessation. The reason is that nicotine can reduce anxiety and relieve stress, and patients that quit smoking may well have a lower improvement in the QoL questionnaires because of the cessation ${ }^{(39)}$.

Although our results are in agreement with the findings of previous studies ${ }^{(11-13)}$ that revealed that smoking is associated with reduced QoL improvement after ESS, nevertheless in contrast to those studies supporting a general reluctance to perform ESS on smoking patients with medically recalcitrant CRS, we showed that both groups reported significant improvement in their daily lives after ESS, not justifying this reluctance ${ }^{(11-13)}$. Some discrepancies with previous studies that demonstrated no significant differences in ESS outcomes in smokers versus non smokers suffering from CRS with regard to QoL results ${ }^{(14-18)}$ could be attributed to the way of selecting smoking and non smoking patients. In a recent study of Briggs et al. ${ }^{(13)}$ that reported worse QoL results after ESS in smokers, a recall bias may have caused the results, as periodic or light smokers might not report smoking on the questionnaire, thus their smoking cohort might have only consisted of heavy smokers. Similarly, in the studies by Das et al. ${ }^{(15,16)}$, who found no difference in results, the reason of not reporting the quantity of smoking in their smoking patient cohort may have caused a bias. Thus, smoking volume heterogeneity between previous study cohorts may explain the variation seen in study results and conclusions in prior studies.

Therefore we understand that the impact of the quantity and duration of smoking habits after ESS has not been sufficiently evaluated and still remains a matter of future studies ${ }^{(1)}$. This is the first study that investigates the impact of the quantity of daily smoking and duration of smoking, expressed in pack-years on QoL outcomes. The answer to this question is of great clinical importance as it can explain most of previous findings. We observed that there was a tendency towards smaller improvement of smokers' QoL as the number of pack-years was increasing. Our results suggest that the duration and quantity of daily smoking certainly affects QoL outcomes after ESS. Moreover, the increase in the number of pack-years in smokers that significantly affects QoL outcomes was quantitatively explored for the first time. Multivariate linear regression analysis on the post-operative values of the QoL questionnaires showed that an increase of 10 pack-years was ensued by a significant deterioration of all QoL questionnaire results by 1.65 up to 12.35 points according to the psychometric instrument used (QOD by 2.00 points; ZAS by 8.28 ; STAI by 9.11 ; ZDS by 8.87 ; BDI by 1.65 ; and SF-36 by 12.35 points). These findings are of great clinical importance in every day clinical practice for better counselling patients and surgical case selection with regard to $\mathrm{Q} o \mathrm{~L}$ results.

In conclusion, CRS patients experience beneficial QoL results after ESS irrespective to their smoking habits. Overall, the longterm results appear to remain very encouraging when patients with good indications for surgery undergo ESS and meticulous postoperative surgical and medical management is performed. Both smokers and non-smokers are significantly improved after ESS and there is no contra-indication to surgery related to smoking habits; however, the improvement of QoL outcomes for non-smokers is significantly higher than in smokers. Additionally, there is a direct association between the quantity and duration of smoking and deterioration in QoL questionnaires' results. An increase of 10 in the number of pack-years is followed by significant worse post-operative QoL results. From a clinical standpoint, this knowledge is very important as it may explain the impact of smoking habits on ESS outcomes with regard to QoL results, and sends the message to doctors to counsel their patients to stop smoking as long as possible prior to surgery as it may further improve treatment outcomes on patients' QoL.

\section{Authorship contribution}

MK designed, conceived and conducted the clinical study, investigated and analyzed the data, wrote article; ES conceived and conducted the clinical study related to psychological issues, investigated and wrote article; GT made all the statistical analysis of the data, and wrote article; NZ conceived the clinical study, analyzed the data, wrote the article and build up the cooperation; GD collected all data (clinical data, questionnaires), participated in the conduction of the clinical study, gave clinical support and wrote the manuscript; PG conducted the clinical 
study, investigated and analyzed the data, wrote article; CB and VD designed, conceived the study, analyzed the data, wrote the article, build up the cooperation and approved the final version for publication.

\section{Conflicts of Interest}

No conflict of interest exists.

\section{References}

1. Reh DD, Higgins TS, Smith TL. Impact of tobacco smoke on chronic rhinosinusitis: a review of the literature. Int Forum Allergy Rhinol. 2012; 2: 362-369.

2. Wu AW, Ting JY, Platt MP, Tierney HT, Metson R. Factors affecting time to revision sinus surgery for nasal polyps: A 25-year experience. Laryngoscope. 2014; 124: 29-33.

3. Goldstein-Daruech N, Cope EK, Zhao KQ et al. Tobacco smoke mediated induction of sinonasal microbial biofilms. PLoS One. 2011; 6: 6 (1).

4. Tamashiro E, Xiong G, Anselmo-Lima WT, Kreindler JL, Palmer JN, Cohen NA. Cigarette smoke exposure impairs respiratory epithelial ciliogenesis. Am J Rhinol Allergy. 2009; 23: 117-122.

5. Katotomichelakis $M$, Balatsouras $D$, Tripsianis G, et al. The effect of smoking on the olfactory function. Rhinology. 2007; 45 273-280.

6. Kennedy DW. Pathogenesis of chronic rhinosinusitis. Ann Otol Rhinol Laryngol Suppl. 2004; 193: 6-9.

7. Reh DD, Lin SY, Clipp SL, Irani L, Alberg AJ, Navas-Acien A. Second hand tobacco smoke exposure and chronic rhinosinusitis: a population-based case-control study. Am J Rhinol Allergy. 2009; 23: 562-567.

8. Houser SM, Keen KJ. The role of allergy and smoking in chronic rhinosinusitis and polyposis. Laryngoscope. 2008; 118: 1521-1527.

9. Senior BA, Kennedy DW, Tanabodee J, Kroger H, Hassab M, Lanza D. Long-term results of functional endoscopic sinus surgery. Laryngoscope. 1998; 108: 151-157.

10. Sugiyama K, Matsuda $T$, Kondo $H$, et al. Postoperative olfaction in chronic sinusitis: smokers versus non smokers. Ann Otol Rhinol Laryngol. 2002; 111: 1054-1058.

11. Danielsen A, Olofsson J. Endoscopic endonasal sinus surgery. A long-term follow-up study. Acta Otolaryngol. 1996; 116: 611-619.

12. Sobol SE, Wright ED, Frenkiel S. One-year outcome analysis of functional endoscopic sinus surgery for chronic sinusitis. J Otolaryngol. 1998; 27: 252-257.

13. Briggs RD, Wright ST, Cordes S, Calhoun KH. Smoking in chronic rhinosinusitis: a predictor of poor long-term outcome after endoscopic sinus surgery. Laryngoscope. 2004; 114: 126-128.

14. Smith TL, Mendolia-Loffredo S, Loehrl TA, Calhoun $\mathrm{KH}$. Predictive factors and outcomes in endoscopic sinus surgery for chronic rhinosinusitis. Laryngoscope. 2005; 115: 2199-2205.

15. Das S, Khichi SS, Perakis H, Woodard T, Kountakis SE. Effects of smoking on quality of life following sinus surgery: 4-year followup. Laryngoscope. 2009; 119: 2284-2287.
16. Das S, Becker AM, Perakis H, Prosser JD, Kountakis SE. The effects of smoking on short-term quality of life outcomes in sinus surgery. Laryngoscope. 2007; 117: 2229 2232.

17. Rudmik L, Mace JC, Smith TL. Smoking and Endoscopic Sinus Surgery: Does smoking volume contribute to clinical outcome? Int Forum Allergy Rhinol. 2011; 1: 145-152.

18. Krzeski A, Galewicz A, Chmielewski R, Kisiel M. Influence of cigarette smoking on endoscopic sinus surgery long-term outcomes. Rhinology. 2011; 49: 577-582.

19. Watelet JB, Annica B, van Cauwenberge P, Bachert C. Objective outcome after functional endoscopic sinus surgery: prediction factors. Laryngoscope. 2004; 114: 1092 1097.

20. Danielides $V$, Katotomichelakis $M$ Balatsouras D, et al. Improvement of olfaction after endoscopic sinus surgery in smokers and nonsmokers. Ann Otol Rhinol Laryngol. 2009; 118: 13-20.

21. Hummel T, Sekinger B, Wolf SR, Pauli E, Kobal G. 'Sniffin' sticks': olfactory performance assessed by the combined testing of odor identification, odor discrimination and olfactory threshold. Chem Senses. 1997; 22: 39-52.

22. Katotomichelakis $M$, Balatsouras $D$, Tripsianis G, Tsaroucha A, Homsioglou E, Danielides V. Normative values of olfactory function testing using the 'Sniffin' Sticks.' Laryngoscope. 2007; 117: 114-120.

23. Lund VJ, and Mackay IS. Staging in rhinosinusitis. Rhinology. 1993; 31: 183-184.

24. Fokkens WJ, Lund VJ, Mullol J, et al. EPOS 2012: European position paper on rhinosinusitis and nasal polyps 2012. A summary for otorhinolaryngologists. Rhinology. 2012; 50: 1-12.

25. Stammberger $\mathrm{H}$. Endoscopic endonasal surgery: concepts in treatment of recurring rhinosinusitis - II: surgical technique. Otolaryngol Head Neck Surg. 1986; 94: 147156.

26. Simopoulos $E$, Katotomichelakis $M$, Gouveris H, Tripsianis G, Livaditis M, Danielides $V$. Olfaction-associated quality of life in chronic rhinosinusitis: Adaptation and validation of an olfaction specific questionnaire. Laryngoscope. 2012; 122: 1450-1454.

27. Zung W. A rating instrument for anxiety disorders. Psychosomatics. 1971; 12: 371-379.

28. Spielberger CD, Gorsuch RL, Lushene R, et al. Manual for the State-Trait Anxiety Inventory. Palo Alto, CA: Consulting Psychologists Press: 1983.

29. Beck AT, Ward CH, Mendelson M, Mock J, Erabaugh J. An Inventory for Measuring Depression. Arch General Psychiatry 1961; 4: 53-63.
30. Fountoulakis KN, lacovides A, Samolis S, et al. Reliability, Validity and Psychometric Properties of the Greek Translation of the Zung Depression Rating Scale. BMC Psychiatry. 2001; $1: 6$.

31. Ware JE. The SF-36 Health Survey. In: Spilker B (eds). Quality of life and pharmaeconomics in clinical trials. Philadelphia, PA. Lipincott-Raven; 1996: 337-346.

32. Pappa E, Kontodimopoulos N, Niakas D. Validating and norming of the Greek SF-36 Health Survey. Qual Life Res. 2005; 14: 14331438.

33. Cohen J. Statistical power analysis for the behavioral sciences. (2nd Ed.) Hillsdale, NJ: Lawrence Erlbaum Associates; 1988.

34. Katotomichelakis M, Simopoulos $E$, Tripsianis G, et al. Predictors of quality of life outcomes in chronic rhinosinusitis after sinus surgery. Eur Arch Otorhinolaryngol. 2014; 271: 733-741.

35. Norman GR, Sloan JA, Wyrwich KW. Interpretation of changes in health-related quality of life: the remarkable universality of half a standard deviation. Med Care 2003; 41: 582-592.

36. Contopoulos-loannidis DG, Karvouni A, Kouri I, et al. Reporting and interpretation of SF-36 outcomes in randomised trials: systematic review. BMJ 2009; 12; 338:a3006.

37. Fisher PL, Durham RC. Recovery rates in generalized anxiety disorder following psychological therapy: an analysis of clinically significant change in the STAI-T across outcome studies since 1990. Psychol Med. 1999; 29: 1425-1434.

38. Steer RA, Cavalieri TA, Leonard DM, et al. Use of the Beck Depression Inventory for Primary Care to screen for major depression disorders. Gen Hosp Psychiatry. 1999; 21: 106-111.

39. Picciotto MR, Brunzell DH, Caldarone BJ. Effect of nicotine and nicotinic receptors on anxiety and depression. Neuroreport. 2002; 13: 1097-1106.

Vassilios Danielides, MD, PhD

Department of Otorhinolaryngology

University Hospital of

Alaxandroupolis

Alexandroupolis 68100

Greece

Tel: $+30-2551074544$

Fax: $+30-2551030441$

E-mail:vdanielidis@hotmail.com 